\title{
Erratum
}

\section{Variable window width kernel estimates of probability densities}

\author{
Peter Hall ${ }^{1}$ and J.S. Marron ${ }^{2}$ \\ ${ }^{1}$ Australian National University, G.P.O. Box 4, Canberra ACT 2601, Australia \\ ${ }^{2}$ University of North Carolina, Chapel Hill, NC 27599-3260, USA
}

Probab. Theory Relat. Fields 80, 37-49 (1988)

Received September 23, 1991

The estimator treated in this paper should be modified by one of a variety of different possible types of truncation, selected to limit the influence of extreme data values while preserving the asymptotic features outlined by Hall and Marron (1988). Similar modifications should be made to the estimators treated by other authors in the context of variable kernel density estimation, if the good convergence rate properties claimed for these estimators are to be ensured. Details of the necessary alterations, including several practical proposals, are given in Hall et al. (1991). That paper shows that the bias of these slightly modified estimators does have the nice property of a bias rate which is the same as higher order kernels.

The susceptibility of the original estimator to unexpected tail effects was pointed out in an example by Scott and Terrell (1991). Hall et al. (1991) also measure the extent of the influence of extreme data values on the unadjusted estimator, under a variety of tail conditions, in terms of an additional term in the representation given in Theorem 2.1 of Hall and Marron (1988).

\section{References}

1. Hall, P., Hu, T.C., Marron, J.S.: Improved variable window kernel estimates of probability densities. North Carolina Institute of Statistics. Mimeo Series \# 2051 (1991)

2. Scott, D.W., Terrell, G.R.: Variable kernel density estimation (unpublished manuscript, 1991) 